\title{
Comparação entre parâmetros de avaliação do estado nutricional e força de preensão palmar em indivíduos com tumores sólidos e hematológicos
}

\author{
Comparison between parameters of nutritional status and handgrip strength in individuals \\ with solid and hematological tumors
}

\section{Comparación entre parámetros para la evaluación del estado nutricional y la fuerza de agarre en individuos con tumores sólidos y hematológicos}

Bruna Luísa Gomes de Miranda ${ }^{1}$, Thalles Marciano de Santana Ferreira ${ }^{1}$, Isabela Naves de Sousa ${ }^{1}$, Ana Beatriz Dantas Mendes ${ }^{1}$, Ana Carolina Lúcio Pereira da Silva ${ }^{2}$, Clélia Carla de Medeiros Carvalho Azevedo², Iasmin Matias de Sousa1, Márcia Marília Gomes Dantas Lopes ${ }^{1 *}$.

\section{RESUMO}

Objetivo: Comparar parâmetros de avaliação nutricional e capacidade funcional em indivíduos com tumores sólidos (TSs) e hematológicos (THs). Métodos: Estudo transversal de amostragem não probabilística, incluindo adultos e idosos com câncer. Dados foram coletados via questionário, consulta ao prontuário, e avaliação do estado nutricional de pacientes no Hospital Universitário Onofre Lopes (HUOL), Natal - RN. Parâmetros avaliados: Índice de Massa Corporal (IMC), Perímetro do Braço (PB), Dobra Cutânea Tricipital (DCT), espessura do Músculo Adutor do Polegar (MAP), capacidade funcional por Força de Preensão Palmar, Avaliação Subjetiva Global Produzida pelo Próprio Paciente (ASG-PPP) e risco de sarcopenia pelo GripBMI. Resultados: Não houve diferenças significativas nos dados descritivos. Indivíduos com TSs apresentaram menores médias em IMC $\left(22,2 \mathrm{~kg} / \mathrm{m}^{2}\right)$, PB $(26,1 \mathrm{~cm})$, DCT $(13,2 \mathrm{~mm})$, e classificações mais comprometidas pela ASG-PPP (B e C). Todos os indivíduos com TSs e $60 \%$ dos com THs apresentaram desnutrição pelo MAP. 37\% dos com TSs apresentaram risco de sarcopenia pelo GripBMI, enquanto nenhum dos com THs apresentou. Conclusão: Indivíduos com TSs apresentam maior comprometimento nutricional comparados aos com THs. Os achados mostram a importância do olhar diferenciado para o cuidado nutricional com relação ao sítio tumoral.

Palavras-chave: Estado nutricional, Neoplasias, Dinamômetro de força muscular, Avaliação nutricional.

\begin{abstract}
Objective: To compare parameters of nutritional assessment and functional capacity in individuals with solid (STs) and hematological tumors (HTs). Methods: Non-probabilistic sampling cross-sectional study, including adult and elderly people with cancer. Data were collected through a questionnaire, consultation of medical records and assessment of nutritional status of patients at the Onofre Lopes University Hospital (OLUH), Natal - RN. Evaluated parameters: Body Mass Index (BMI), Arm Circumference (AC), Tricipital Skinfold (TS), Adductor Pollicis Muscle (APM) thickness, functional capacity by Hand Grip Strength, Patient-Generated Subjective Global Assessment (PG-SGA), and risk of sarcopenia by GripBMI. Results: There were no significant differences in the descriptive data. Individuals with STs had lower averages in $B M I\left(22,2 \mathrm{~kg} / \mathrm{m}^{2}\right), A C$ $(26,1 \mathrm{~cm})$, TS $(13,2 \mathrm{~mm})$, and more compromised classifications by PG-SGA (B and C). All individuals with STs and $60 \%$ of those with HTs showed malnutrition by APM. $37 \%$ of those with STs were at risk of sarcopenia by GripBMI, while none of those with HTs did. Conclusion: Individuals with STs had a greater nutritional
\end{abstract}

1 Universidade Federal do Rio Grande do Norte (UFRN), Natal - RN. *E-mail: mariliagdantas@hotmail.com

2 Hospital Universitário Onofre Lopes (HUOL), Natal - RN. 
impairment compared to those with HTs. The findings show the importance of a differentiated look for nutritional care in relation to the tumor site.

Keywords: Nutritional status, Neoplasms, Muscle strength dynamometer, Nutritional assessment.

\section{RESUMEN}

Objetivo: Comparar parámetros de valoración nutricional y capacidad funcional en individuos con tumores sólidos (TSs) y hematológicos (THs). Métodos: Estudio transversal de muestreo no probabilístico, incluyendo adultos y ancianos con cáncer. Datos obtenidos mediante cuestionario, consulta de historias clínicas y evaluación del estado nutricional de pacientes en Hospital Universitario Onofre Lopes (HUOL), Natal - RN. Parámetros evaluados: Índice de Masa Corporal (IMC), Circunferencia del Brazo (CB), Pliegue Cutáneo Tricipital (PCT), espesor del Músculo Aductor del Pulgar (MAP), capacidad funcional mediante Fuerza de Prensión Manual, Evaluación Subjetiva Global Producida por Propio Paciente (ESG-PPP) y riesgo de sarcopenia por GripBMI. Resultados: No hubo diferencias significativas en datos descriptivos. Individuos con TS tenían promedios más bajos en IMC $\left(22,2 \mathrm{~kg} / \mathrm{m}^{2}\right)$, CB $(26,1 \mathrm{~cm})$, PCT $(13,2 \mathrm{~mm})$ y clasificaciones más comprometidas según ESG-PPP ( $B$ y $C$ ). Todos individuos con TSs y $60 \%$ de aquellos con THs mostraron desnutrición por MAP. 37\% de las personas con TSs estaban en riesgo de sarcopenia por GripBMI, mientras que ninguna de las personas con TSs lo hizo. Conclusión: Los individuos con TSs tienen un mayor deterioro nutricional en comparación con aquellos con THs. Los hallazgos muestran la importancia de una mirada diferenciada para el cuidado nutricional en relación al sitio del tumor.

Palabras clave: Estados nutricionales, Neoplasias, Dinamómetro de fuerza muscular, Valoración nutricional.

\section{INTRODUÇÃO}

O câncer é caracterizado pelo crescimento desordenado de células, que invadem tecidos e órgãos. Estas, proliferam-se rapidamente, determinando a formação de tumores malignos, que podem espalhar-se para outras regiões do corpo (MINISTÉRIO DA SAÚDE, 2016). Configura-se como uma doença crônica não transmissível com etiologia multifatorial, que interage com fatores genéticos, ambientais e de estilo de vida, e está entre as principais causas de morte no mundo (STEEMBURGO T, et al., 2018). Segundo o Instituto Nacional de Câncer José Alencar Gomes da Silva (INCA), para o Brasil, a estimativa para cada ano do triênio 2020-2022 aponta a ocorrência de 625 mil novos casos de câncer (excluindo os casos de câncer de pele não melanoma) (MINISTÉRIO DA SAÚDE, 2019).

Dentre as consequências desta doença é comum o déficit nutricional, e está associado ao aumento da morbimortalidade, principalmente devido à anorexia, ativação do sistema inflamatório, alterações no metabolismo e aumento do gasto de energia. Todos esses fatores combinados levam a uma perda de peso acentuada e redução da capacidade funcional (STEEMBURGO T, et al., 2018). Dessa forma, a desnutrição configura um impacto nutricional frequentemente presente em pacientes com câncer, sendo esta uma desnutrição calórico-proteica, que ocorre devido a um desequilíbrio entre a ingestão e as necessidades nutricionais desses pacientes (CAGOL F, et al., 2016).

Além disso, é grande a associação do estado nutricional sobre o prognóstico e o tratamento, de modo que a agressividade, a localização do tumor, os órgãos envolvidos, as condições clínicas e imunológicas, o diagnóstico tardio e a magnitude da terapêutica contribuem diretamente para o agravo nutricional (SOCIEDADE BRASILEIRA DE NUTRIÇÃO ONCOLÓGICA (SBNO), 2021).

Sabe-se que cerca de $80 \%$ dos pacientes com câncer apresenta desnutrição já no momento do diagnóstico, e além disso, $20 \%$ dos óbitos nesses pacientes ocorre devido a complicações decorrentes da desnutrição e não da doença propriamente dita; portanto, a avaliação e acompanhamento nutricional são fundamentais, visando reduzir o risco de alterações nutricionais decorrentes da doença e do tratamento (CAGOL F, et al., 2016; LIMBERGER VR, et al., 2014). 
Nesse sentido, algumas das abordagens frequentemente utilizadas em ambiente hospitalar para a avaliação nutricional do paciente oncológico, são as medidas de avaliação antropométrica. Estas, são de fácil aplicabilidade, baixo custo, pouco invasivas, de fácil execução e padronização, aplicáveis a todas as faixas etárias e cursos da vida e visam avaliar a composição corporal, contribuindo para um diagnóstico nutricional mais preciso (SBNO, 2021).

Considerando as diferentes origens dos tumores, os tumores sólidos (TSs) são caracterizados por um aglomerado de células neoplásicas, formado em decorrência de um crescimento anormal de células de um tecido. Já os tumores hematológicos (THs), incluem um grupo de doenças malignas que afetam os precursores hematopoiéticos da medula óssea e que não estão restritas a uma única região do corpo (ALVES ASBM, et al., 2018).

Nesse panorama, estudos apontam que indivíduos com THs possuem um estado nutricional diferente daqueles com TSs, caracterizado por complicações nutricionais não tão graves, quando comparadas às existentes no perfil nutricional de indivíduos com TSs (BARATA AT, et al., 2017; LETILOVIC T, et al., 2017). Tal achado pode ser observado ao comparar os estudos de Rodrigues BC, et al. (2019), que ao avaliar pacientes com THs hospitalizados verificou apenas 8,7\% dos pacientes com Índice de Massa Corporal (IMC) na classificação de desnutrição, enquanto Lima JS, et al. (2018), que avaliou pacientes com TSs gastrointestinais, observou este diagnóstico nutricional em $43,9 \%$ dos indivíduos.

Outro aspecto importante na complementação desta avaliação é a força de preensão palmar (FPP), que determina a capacidade funcional e detecta alterações funcionais em curtos períodos de tempo (MINISTÉRIO DA SAÚDE, 2016; LIMBERGER VR, et al., 2014; STEEMBURGO T, et al., 2018). É um parâmetro importante, pois a perda de massa magra, força e da capacidade funcional, estão diretamente relacionados à progressão da doença, piora do quadro clínico e declínio do estado nutricional (VALENTE KP, et al., 2019).

Diante dos dados alarmantes sobre a crescente incidência e mortalidade de pacientes com câncer, é fundamental o desenvolvimento de estudos que investiguem métodos de avaliação do estado nutricional e capacidade funcional a fim de obter uma avaliação fidedigna do paciente a curto prazo e da resposta da terapia nutricional aplicada. Com isso, o presente estudo tem por objetivo comparar parâmetros de avaliação nutricional e força e preensão palmar em indivíduos com diferentes sítios tumorais.

\section{MÉTODOS}

O estudo foi aprovado pelo Comitê de Ética em Pesquisa do Hospital Universitário Onofre Lopes (HUOL) (parecer n. 2.404.828), e foi realizado nas enfermarias do HUOL. Após explicação detalhada acerca do estudo, o paciente foi convidado a participar e sua participação foi validada a partir da assinatura do Termo de Consentimento Livre e Esclarecido em duas vias.

O estudo foi do tipo transversal, com amostragem não probabilística, realizado entre agosto de 2019 e março de 2020. Foram incluídos indivíduos adultos e idosos, com idade superior a 20 anos e com diagnóstico de câncer. Pacientes em uso de corticoterapia foram excluídos.

A coleta de dados ocorreu de forma prospectiva a partir da aplicação de questionário pré-elaborado e consulta ao prontuário para coletar informações de idade, sexo, sítio tumoral e modalidade de tratamento.

A avaliação antropométrica compreendeu a mensuração do peso corporal, altura, Perímetro do Braço (PB), Dobra Cutânea Tricipital (DCT) e Músculo Adutor do Polegar (MAP). A partir dos valores de peso e altura, o IMC foi calculado e classificado de acordo com os valores de referência estabelecidos para adultos, e para idosos (indivíduos com idade maior ou igual a 60 anos) (WORLD HEALTH ORGANIZATION (WHO), 1995; LIPSCHITZ DA, 1994).

As medidas de peso e altura foram realizadas, respectivamente, em balanças calibradas e estadiômetros fixos. Os voluntários foram avaliados descalços e com roupas leves, e despidos de qualquer adorno que pudesse interferir na aferição para mensuração. Foram posicionados no centro da plataforma, em postura ereta, com os pés juntos, braços estendidos e mãos ao lado do corpo. 
O PB foi avaliado com o braço do paciente flexionado e posicionado ao lado do tórax, formando um ângulo de $90^{\circ}$ para localização do ponto médio entre o acrômio e o olecrano. A aferição foi realizada com fita flexível no ponto marcado, de forma ajustada e evitando compressão da pele, como preconizado por Kamimura MA, et al. (2014), e os valores obtidos foram classificados de acordo com Frisancho AR (1990).

Utilizando-se o adipômetro Lange (Beta Technology Incorporated, Cambridge, Maryland, USA) foram aferidas DCT e MAP. Para a aferição da DCT, o indivíduo avaliado manteve o braço esticado e com a musculatura relaxada, e a dobra foi medida na sua face dorsal, sob o tríceps, no ponto médio entre o acrômio e o olecrano. Os parâmetros definidos por Frisancho AR (1990) foram utilizados para a classificação da medida em questão.

O MAP foi aferido com o indivíduo sentado, com a mão dominante apoiada no joelho. A aferição foi feita no vértice de um triângulo imaginário formado pela extensão do polegar e do dedo indicador, de acordo com a técnica estabelecida na literatura (LAMEU EB, et al., 2004). Tendo em vista que não há ponto de corte definido para pacientes com câncer, foi utilizado o ponto de corte para pacientes cirúrgicos de acordo com Bragagnolo R, et al. (2009), o qual define que valores menores que 13,4 mm indicam desnutrição.

A Avaliação Subjetiva Global Produzida pelo Próprio Paciente (ASG-PPP) foi aplicada pelo pesquisador diretamente ao paciente, e incluiu questões sobre alteração de peso, ingestão alimentar, sintomas relacionados ao câncer e alteração da capacidade nutricional. Para este estudo foi utilizada a versão traduzida e validada para o português, mediante permissão de uso da ASG-PPP/Pt-Global Platform (www.pt-global.org) (SILVA S e PINHO J, 2015). A avaliação do percentual de perda de peso foi feita de acordo com a tabela de significado da perda de peso em relação ao tempo (BLACKBURN GL, et al., 1977). Foram realizados também, exame físico e avaliação de alterações que aumentam a demanda metabólica. Por fim, o pesquisador determinou o diagnóstico nutricional do paciente: bem nutrido (ASG-PPP A), desnutrição moderada ou suspeita de desnutrição (ASG-PPP B) ou desnutrição grave (ASG-PPP C).

A avaliação da capacidade funcional, mediante FPP, foi realizada com auxílio do dinamômetro hidráulico de mão JAMAR (SH5001 Saehan). Para obtenção do valor, foi levado em consideração a avaliação somente da mão dominante, com o paciente sentado, cotovelo flexionado a $90^{\circ}$ e antebraço em posição neutra. Esse, foi orientado a realizar três momentos de máxima contração muscular, com duração aproximada de três segundos, e intervalo de descanso entre 10 a 60 segundos entre cada um destes. Para determinação do valor final, foi utilizado o maior valor obtido entre as aferições, de acordo com o recomendado pela literatura (SCHLÜSSEL MM, et al., 2008), e para fins de comparação, foram utilizados os pontos de corte preconizados para diagnóstico de sarcopenia, que estabelecem valores $<27 \mathrm{~kg}$ para homens e $<16 \mathrm{~kg}$ para mulheres como de baixa FPP (CRUZ-JENTOFT AJ, et al., 2019).

Para a avaliação do risco de sarcopenia, foi utilizada a ferramenta de triagem GripBMI, utilizando os parâmetros de FPP e IMC. Para fins de classificação, a combinação de baixos valores de FPP (menor que $27 \mathrm{Kg}$ para homens e que $16 \mathrm{Kg}$ para mulheres) e IMC de valor menor ou igual a $25 \mathrm{~kg} / \mathrm{m}^{2}$ configura um teste de rastreamento positivo para sarcopenia (CHURILOV I, et al., 2020).

A análise estatística foi realizada utilizando-se o pacote estatístico Statistical Package for the Social Sciences (SPSS) versão 22.0. A normalidade da distribuição dos dados foi realizada utilizando o teste Shapiro-Wilk. Dados quantitativos estão apresentados em média e desvio padrão e os categóricos expressos como números absolutos e frequências relativas. As variáveis quantitativas foram comparadas utilizando o teste t para amostras independentes, e para as variáveis categóricas ou possíveis associações, foi utilizado o teste Qui-quadrado ou exato de Fisher. O nível de significância adotado para todas as análises foi de 5\%.

\section{RESULTADOS}

Os dados descritivos da amostra estão apresentados na Tabela 1 de acordo com o tipo de tumor. A maioria dos participantes eram adultos $(61,9 \%)$, e acometidos por TSs $(76,2 \%)$, sendo $88 \%$ no trato gastrointestinal (estômago, vias biliares, pâncreas, fígado e cólon) e $12 \%$ do trato urinário. O tratamento quimioterápico foi o mais frequente, e nenhum dos pacientes avaliados estava em tratamento de radioterapia, imunoterapia ou 
cuidados paliativos. Ao comparar os dados descritivos da amostra com relação ao tipo de tumor, não foram obtidas diferenças significativas. Dessa forma, os dados foram considerados homogêneos para algum viés que pudesse ter influenciado nas características do estado nutricional ou de capacidade funcional.

Tabela 1 - Dados descritivos da amostra de pacientes com TSs e THs, $n=21$.

\begin{tabular}{lcccc}
\hline Variável & Total $(\mathbf{n}=\mathbf{2 1})$ & Sólidos $(\mathbf{n}=\mathbf{1 6})$ & Hematológicos $(\mathbf{n = 5 )}$ & $\mathbf{p}$ \\
\hline Idade (média \pm DP) & $53,0 \pm 15,7$ & $56,1 \pm 15,0$ & $44,6 \pm 16,0$ & 0,158 \\
Idosos $(\mathrm{n}, \%)$ & $8(38,1)$ & $8(50,0)$ & $0(0)$ & 0,111
\end{tabular}

\section{Sexo}

\begin{tabular}{lllll}
\hline Masculino (n, \%) & $11(52,4)$ & $7(43,8)$ & $4(80,0)$ & 0,311 \\
Feminino (n, \%) & $10(47,6)$ & $9(56,2)$ & $1(20,0)$ & \\
\hline
\end{tabular}

\section{Hábitos de vida}

\begin{tabular}{lcccc}
\hline Etilismo/Ex etilista (n, \%) & $13(61,9)$ & $10(62,5)$ & $3(60,0)$ & 1,000 \\
Tabagismo/Ex tabagista (n, \%) & $8(38,1)$ & $7(43,8)$ & $1(20,0)$ & 0,606 \\
\hline Comorbidades & & & & \\
\hline HAS (n, \%) & $7(33,3)$ & $5(31,3)$ & $2(40,0)$ & 1,000 \\
Cardiopatias (n, \%) & $3(14,3)$ & $2(12,5)$ & $1(20,0)$ & 1,000 \\
DM (n, \%) & $3(14,3)$ & $3(18,8)$ & $0(0)$ & 0,549 \\
Dislipidemia (n, \%) & $2(9,5)$ & $2(12,5)$ & $0(0)$ & 1,000 \\
Renal (n, \%) & $2(9,5)$ & $2(12,5)$ & $0(0)$ & 1,000 \\
\hline
\end{tabular}

\section{Tratamento realizado}

\begin{tabular}{lcccc}
\hline QT $(\mathrm{n}, \%)$ & $15(71,4)$ & $11(68,8)$ & $4(80,0)$ & 1,000 \\
Cirurgia (n, \%) & $6(28,6)$ & $5(31,3)$ & $1(20,0)$ & 1,000 \\
\hline Tempo de diagnóstico & & & \\
\hline Maior que 6 meses (n, \%) & $11(52,4)$ & $9(56,2)$ & $2(40,0)$ & 0,635 \\
Menor que 6 meses (n, \%) & $10(47,6)$ & $7(43,8)$ & $3(60,0)$ &
\end{tabular}

Legenda: TSs: Tumores Sólidos. THs: Tumores Hematológicos. DP: Desvio Padrão. HAS: Hipertensão Arterial Sistêmica. DM: Diabetes Mellitus. QT: Quimioterapia. Nota: Dados apresentados em média e desvio padrão, frequência absoluta e relativa; Valores de $p$ obtidos pelo teste t para amostras independentes, Quiquadrado ou Exato de Fisher. Fonte: Miranda BLG, et al., 2022.

As características de avaliação do estado nutricional são observadas na Tabela 2. A partir desta, é possível observar menores médias nos valores de IMC, PB e MAP nos participantes com TSs, bem como maior percentual de indivíduos com diagnóstico de desnutrição (pelo MAP) e baixo peso (pelo IMC) em comparação ao grupo dos THs. 
Tabela 2 - Características do estado nutricional e força de preensão palmar de pacientes de acordo com o tipo de câncer, $\mathrm{n}=21$.

\begin{tabular}{|c|c|c|c|c|}
\hline Variável & Total $(\mathbf{n}=21)$ & Sólidos ( $n=16$ ) & Hematológicos $(n=5)$ & $p$ \\
\hline IMC (média \pm DP) ${ }^{\mathrm{a}}$ & $23,4 \pm 4,0$ & $22,2 \pm 3,8$ & $27,2 \pm 1,7$ & 0,012 \\
\hline Baixo peso & $7(33,3 \%)$ & $7(43,8 \%)$ & $0(0 \%)$ & 0,081 \\
\hline Eutrofia & $6(28,6 \%)$ & $6(37,5 \%)$ & $0(0 \%)$ & \\
\hline Sobrepeso & $8(38,1 \%)$ & $3(18,8 \%)$ & $5(100 \%)$ & \\
\hline PB (média \pm DP) ${ }^{b}$ & $27,4 \pm 5,0$ & $26,1 \pm 4,8$ & $31,5 \pm 3,4$ & 0,032 \\
\hline Baixo peso & $6(28,6 \%)$ & $6(37,5 \%)$ & $0(0)$ & \multirow{4}{*}{0,036} \\
\hline Abaixo da média & $1(4,8 \%)$ & $1(6,25 \%)$ & $0(0)$ & \\
\hline Adequado & $11(52,4 \%)$ & $7(43,8 \%)$ & $4(80,0 \%)$ & \\
\hline Acima da média & $1(4,8 \%)$ & $0(0)$ & $1(20,0 \%)$ & \\
\hline 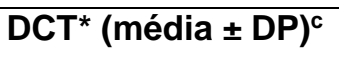 & $14,2 \pm 5,5$ & $13,2 \pm 5,6$ & $17,6 \pm 4,2$ & 0,122 \\
\hline Magreza (depleção) & $4(19,1 \%)$ & $4(25,0 \%)$ & $0(0)$ & \multirow{4}{*}{0,086} \\
\hline Abaixo da média & $2(9,5 \%)$ & $2(12,5 \%)$ & $0(0)$ & \\
\hline Adequado & $10(47,6 \%)$ & $7(43,8 \%)$ & $3(60,0 \%)$ & \\
\hline Acima da média & $3(14,3 \%)$ & $1(6,25 \%)$ & $2(40,0 \%)$ & \\
\hline MAP (média \pm DP) ${ }^{d}$ & $8,7 \pm 3,8$ & $7,3 \pm 2,7$ & $12,9 \pm 3,9$ & 0,002 \\
\hline Desnutrição & $19(90,5 \%)$ & $16(100 \%)$ & $3(60 \%)$ & \multirow{2}{*}{0,048} \\
\hline Sem desnutrição & $2(9,5 \%)$ & $0(0 \%)$ & $2(40 \%)$ & \\
\hline
\end{tabular}

\begin{tabular}{lcccc}
\hline ASG-PPP & & & & \\
\hline Escore (média \pm DP) & $10,5 \pm 5,0$ & $10,7 \pm 4,6$ & $10,0 \pm 6,7$ & 0,797 \\
A & $12(57,1 \%)$ & $8(50,0 \%)$ & $4(80,0 \%)$ & \\
B & $7(33,3 \%)$ & $6(37,5 \%)$ & $1(20,0 \%)$ & 0,770 \\
C & $2(9,5 \%)$ & $2(12,5 \%)$ & $0(0 \%)$ & \\
\hline FPP (média \pm DP) & $21,2 \pm 8,2$ & $21,4 \pm 9,3$ & $20,7 \pm 3,8$ & 0,878 \\
\hline FPP normal & $9(42,9 \%)$ & $9(56,2 \%)$ & $0(0 \%)$ & \multirow{2}{*}{0,045} \\
FPP baixa & $12(57,1 \%)$ & $7(43,8 \%)$ & $5(100 \%)$ & \\
\hline GripBMI & & & & \\
\hline Risco de sarcopenia & $6(28,6 \%)$ & $6(37,5 \%)$ & $0(0 \%)$ & 0,262 \\
Sem risco de sarcopenia & $15(71,4 \%)$ & $10(62,5 \%)$ & $5(100 \%)$ & \\
\hline
\end{tabular}

Legenda: DP: Desvio Padrão, IMC: Índice de Massa Corporal, PB: Perímetro do Braço, DCT: Dobra Cutânea Tricipital, MAP: Musculo Adutor do Polegar, ASG-PPP: Avaliação Subjetiva Global Preenchida Pelo Paciente, FPP: Força de Preensão Palmar, GRIP-BMI: ferramenta de triagem de sarcopenia. Nota: Dados apresentados em média e desvio padrão e números absolutos e relativos. Valores de $\mathrm{p}$ obtidos pelo teste t para amostras independentes, Qui-quadrado ou Exato de Fisher. * 2 pacientes não puderam ser classificados por apresentarem idade maior que 74 anos, faixa etária máxima adotada nos percentis utilizados (FRISANCHO AR, 1990). ${ }^{a}$ Adultos: $<18,5 \mathrm{~kg} / \mathrm{m}^{2}$ : baixo peso; 18,5 - 24,9 kg/m²: eutrofia; $>24,9 \mathrm{~kg} / \mathrm{m}^{2}$ : sobrepeso (WHO, 1995). Idosos: $<22,0 \mathrm{~kg} / \mathrm{m}^{2}$ : baixo peso; 22,0-27,0 kg/m²: eutrofia; >27,0 kg/m²: sobrepeso (LIPSCHITZ DA, 1994). ${ }^{\text {b }}$ Percentis: <P5: baixo peso; P5 - P15: abaixo da média; P15 - P85: adequado; P85 - P95: acima da média (FRISANCHO AR, 1990). ${ }^{\circ}$ Percentis: <P5: magreza (depleção); P5 - P15: abaixo da média; P15 - P85: adequado; P85 - P95: acima da média (FRISANCHO AR, 1990). ${ }^{d}<13,4 \mathrm{~mm}$ : desnutrição (BRAGAGNOLO R, et al., 2009). ${ }^{\mathrm{e}}<27 \mathrm{~kg}$ para homens e $<16 \mathrm{~kg}$ para mulheres - baixa FPP (CRUZ-JENTOFT AJ, et al., 2019). ${ }^{\dagger}$ FPP $<27 \mathrm{~kg}$ para homens e $<16 \mathrm{~kg}$ para mulheres + IMC $\leq 25,0$ $\mathrm{kg} / \mathrm{m}^{2}$ - rastreamento positivo para sarcopenia (CHURILOV I, et al., 2020). Fonte: Miranda BLG, et al., 2022. 
Em pacientes com TSs também foi observado maior acometimento do estado nutricional por meio da ASGPPP, com prevalência de $50 \%$ dos pacientes classificados em B ou C, enquanto entre os pacientes com THs, $80 \%$ destes foram classificados na categoria A. Nesse sentido, as diferenças no acometimento do estado nutricional de cada grupo representam um achado pertinente, apesar de não ter sido observada diferença estatística entre as classificações do parâmetro. Em contrapartida, no grupo de indivíduos com TSs foi verificada maior prevalência de valores normais de FPP em comparação ao grupo com THs, no qual todos os pacientes apresentaram baixa FPP. É possível observar que quase $40 \%$ dos pacientes com TSs apresentaram risco de sarcopenia pelo GripBMI, entretanto, o pequeno tamanho amostral de pacientes com THs pode ter influenciado a não observação de diferença entre os grupos.

\section{DISCUSSÃO}

No presente estudo, foram avaliados parâmetros de avaliação do estado nutricional e capacidade funcional em indivíduos com câncer acometidos por diferentes tipos tumorais (sólidos e hematológicos). Os achados mostram que pacientes com TSs apresentam estado nutricional mais comprometido quando comparados aos pacientes com THs.

A avaliação do estado nutricional, pelo IMC, verificou que pacientes com TSs apresentaram menor média em relação aos com THs, já na classificação, a maioria dos indivíduos com TSs apresentou eutrofia ou sobrepeso. Isso demonstra a necessidade de se ter cautela no uso do IMC para avaliar o estado nutricional de pacientes com câncer, já que a desnutrição como um impacto frequentemente observado nessa população.

Comparando o estado nutricional entre os dois grupos estudados, pacientes acometidos por THs apresentaram total prevalência na classificação de sobrepeso pelo IMC, e da mesma forma, os resultados obtidos via ASG-PPP mostram a distribuição desse grupo nas classificações A e B da avaliação, sendo majoritária na primeira categoria. Corroborando esses achados que revelam um estado nutricional mais preservado, em estudo realizado por Valmorbida $A$, et al. (2019) que analisou o estado nutricional de pacientes onco-hematológicos, foi identificado que $56 \%$ dos indivíduos avaliados foram classificados como eutróficos pelo IMC.

Em análise semelhante feita por Rodrigues BC, et al. (2019), foi verificado IMC médio de $26,2 \mathrm{~kg} / \mathrm{m}^{2}$, cuja classificação abrange a faixa de sobrepeso para adultos e de eutrofia para idosos. Assim, o fato de resultados semelhantes terem sido verificados em diferentes parâmetros (IMC e ASG-PPP) pode sugerir que, de fato, esse tipo tumoral pode não apresentar impactos tão agressivos no estado nutricional dos pacientes.

Comparativamente, a maioria dos pacientes com TSs apresentou pior classificação na ASG-PPP. Nessa discussão, acredita-se que THs não estão associados a complicações nutricionais tão graves quando os TSs, que estão relacionados a maiores riscos de desnutrição, sobretudo em razão de que, normalmente, esses sítios tumorais exigem intervenções cirúrgicas; de forma que, o trauma cirúrgico produz como respostas metabólicas no organismo o aumento da perda proteica, desencadeada pela lesão inicial e perpetuada por complicações infecciosas, jejum e imobilidade, além do aumento do metabolismo energético, os quais impactam direta e negativamente o estado nutricional do paciente (BOTTONI A, et al., 2019; MINISTÉRIO DA SAÚDE, 2015).

Além disso, Vale IAV, et al. (2015), em estudo que avaliou a necessidade de intervenção nutricional em pacientes com câncer de diferentes sítios tumorais, segundo o score numérico da ASG-PPP, observou maior necessidade de intervenção nos pacientes com sítios tumorais que interferem com a ingestão/digestão/absorção alimentar, como cabeça e pescoço e tumores do trato gastrointestinal (TSs).

As avaliações de PB e DCT mostraram valores médios ligeiramente menores em pacientes com TSs em relação aos pacientes com THs, além da prevalência de baixo peso pelo PB no primeiro grupo, o que sugere um maior acometimento do estado nutricional nos indivíduos com TSs. Os valores médios de PB e DCT do grupo dos THs foram, maior e muito semelhante, respectivamente, aos de 29,2 cm para PB, analisados por Rodrigues BC, et al. (2019), e de de 18,3 mm para DCT, analisados por Aguiar APN, et al. (2018), ambos estudos que avaliaram pacientes com THs. Por outro lado, ao comparar com os achados de $24 \mathrm{~cm}$ para PB e 13,3 mm para DCT, observados por em indivíduos com câncer de estômago por Poziomyck AK, et al. (2018), 
é possível observar que os valores médios achados no presente estudo para pacientes com THs são bem mais elevados, o que corrobora com a hipótese anteriormente citada de que, em casos de TSs, há um maior comprometimento do estado nutricional.

Em relação ao MAP, o presente estudo verificou que pacientes com TSs apresentaram menores valores médios e maior prevalência de desnutrição pelo MAP. Apesar de o valor médio entre indivíduos com TSs ter sido significativamente menor, em ambos os grupos os valores encontrados estão abaixo da referência adotada, indicando desnutrição (BRAGAGNOLO R, et al., 2009). Outros estudos que avaliaram a espessura do MAP em pacientes com câncer obtiveram valores médios de 13,2 $\mathrm{mm}$ para TSs e 11,9 mm para THs (AGUIAR APN, et al., 2018; POZIOMYCK AK, et al., 2018).

Adicionalmente, a sensibilidade do método é um dos fatores pelos quais muitos estudos abordam a espessura do MAP como importante parâmetro preditor de desnutrição e mortalidade em pacientes com câncer (PAULA ALB, et al., 2018; POZIOMYCK AK, et al., 2017, 2018). Nesse sentido, é válido citar a necessidade da padronização da técnica de aferição desse parâmetro, em vista de diminuir a chance de erros na avaliação, e tornar os resultados obtidos mais fidedignos e o método mais reprodutível.

Em se tratando da avaliação de força e capacidade funcional, a prevalência de baixa FPP entre os indivíduos avaliados se assemelha ao observado em estudo por Maurina ALZ, et. al. (2020) com pacientes com câncer de diferentes sítios tumorais (57\%). A despeito das inúmeras vantagens do método, é válido citar que ainda não há pontos de corte pré-estabelecidos para pacientes com câncer, ou padronização da técnica de aferição, de forma que tais vieses podem afetar a comparação de resultados.

Ainda nesse panorama, sabe-se que a combinação de fatores inerentes ao déficit nutricional pode levar à sarcopenia, uma condição não incomum em pacientes com câncer. Nessa discussão, o GripBMI surge como uma ferramenta que pode ajudar a promover a detecção precoce e o manejo da sarcopenia, e se trata de um instrumento de fácil e rápida execução que utiliza como parâmetros a FPP, que preserva a avaliação da força muscular, e o IMC, que é atribuído como indicador de massa magra (CHURILOV I, et al., 2020).

Como resultado do seu uso na população avaliada no presente trabalho, foi verificado que porção considerável dos pacientes com TSs se encontravam em risco de sarcopenia, enquanto nenhum dos pacientes com THs apresentou tal risco, embora não haja diferença estatística para tal achado. Os critérios utilizados fazem do GripBMI a ferramenta que mais de perto representa os critérios originais utilizados no diagnóstico de sarcopenia pelo Consenso Europeu, de modo que os achados citados sugerem que indivíduos acometidos por TSs apresentam maior risco de desenvolvimento de sarcopenia em comparação com os acometidos por THs.

Em adição, é válido citar a situação mundial atual como um fator limitante no desenvolvimento do estudo em questão. A pandemia do COVID-19 afetou o mundo inteiro, e a coleta de dados precisou ser interrompida antes do previsto, o que prejudicou o tamanho amostral do trabalho. Entretanto, mesmo com o $\mathrm{n}$ amostral limitado, obtivemos resultados que demonstram contribuição científica interessante. Nesse panorama, as análises realizadas não mostraram diferenças estatísticas entre os dados descritivos dos grupos estudados - TSs e THs, ou seja, os resultados encontrados acerca do estado nutricional dos indivíduos não sofreram influência das variáveis de caracterização da amostra, mas foram determinados pelas próprias características nutricionais.

\section{CONCLUSÃO}

Como conclusão, foi possível constatar que as características nutricionais analisadas e os resultados encontrados são sugestivos de que indivíduos com câncer acometidos por TSs sofrem maior impacto no estado nutricional em relação aos acometidos por THs. Além disso, é importante citar a necessidade de avanço acadêmico e desenvolvimento de novos estudos na área, com o objetivo de estabelecer pontos de corte específicos para a população estudada, promover a padronização de técnicas antropométricas e desenvolvimento de ferramentas para facilitar o rastreamento de complicações nutricionais provocados pelas neoplasias de diferentes sítios tumorais e assim, estabelecer estratégias e terapias específicas, a fim de prevenir o agravamento do estado nutricional e promover melhora no quadro clínico. 


\section{REFERÊNCIAS}

1. AGUIAR APN, et al. Adductor pollicis muscle thickness: A sensitive method in the detection of nutritional risk in oncohematological patients. Nutricion Clinica y Dietetica Hospitalaria, 2018; 38(4): 183-188.

2. ALVES ASBM, et al. Epidemiological and cytogenetic profiles of patients with hematological malignancies and their relationship with aging. Hematology, Transfusion and Cell Therapy, 2018; 40(3): 200-206.

3. BARATA AT, et al. Handgrip Dynamometry and Patient-Generated Subjective Global Assessment in Patients with Nonresectable Lung Cancer. Nutrition and Cancer, 2017; 69(1): 154-158.

4. BLACKBURN GL, et al. Nutrition in the Critically III Patient. Anesthesiology, 1977; 47(2): 181-194.

5. BOTTONI A, et al. Cirurgia e Trauma. In: Nutrição Clínica no Adulto. 4. ed. Barueri: Manole, 2019; p. 602.

6. BRAGAGNOLO R, et al. Espessura do músculo adutor do polegar: um método rápido e confiável na avaliação nutricional de pacientes cirúrgicos. Revista do Colegiado Brasileiro de Cirurgiões, 2009; 36(5): 371-376.

7. CAGOL F, et al. Estado nutricional segundo avaliação subjetiva global produzida pelo paciente de acordo com a localização do tumor. Nutricion Clinica y Dietetica Hospitalaria, 2016; 36(4): 13-19.

8. CHURILOV I, et al. GripBMI - A fast and simple sarcopenia screening tool in post acute inpatient rehabilitation. Clinical Nutrition, 2020; 40(3): 1022-1027.

9. CRUZ-JENTOFT AJ, et al. Sarcopenia: revised European consensus on definition and diagnosis. Age and Ageing, 2019; 48(1): 16-31.

10. FRISANCHO AR. Anthropometric Standards for the Assessment of Growth and Nutritional Status. Ann Arbor: University of Michigan Press, 1990; 200 p.

11. KAMIMURA MA, et al. Avaliação Nutricional. In: Nutrição Clínica no Adulto. 3. ed. Barueri: Manole, 2014 ; p. 599.

12. LAMEU EB, et al. The thickness of the adductor pollicis muscle reflects the muscle compartment and may be used as a new anthropometric parameter for nutritional assessment. Current Op in Cli Nut \& Met Care, 2004; 7(3): $293-301$.

13. LETILOVIC T, et al. Anthropometric and Laboratory Variables related to Weight loss - comparison of heart Failure Patients with Tumor Patients and control Population. Frontiers in Nutrition, 2017; 4(18): 3-8.

14. LIMA JS, et al. Avaliação do estado nutricional de pacientes com câncer em um hospital da cidade de Belém - PA. Braspen Journal, 2018; 33(2): 166-70.

15. LIMBERGER VR, et al. Associação entre Dinamometria Manual , Estado Nutricional e Complicações Pós-Operatórias em Pacientes Oncológicos. Revista Brasileira de Cancerologia, 2014; 60(2): 135-141.

16. LIPSCHITZ DA. Screening for nutritional status in the elderly. Primary Care, 1994; $21(1): 55-67$.

17. MAURINA ALZ, et al. Avaliação Nutricional e Funcional em Oncologia e Desfecho Clínico em Pacientes da Cidade de Caxias do Sul/RS. Revista Brasileira de Cancerologia, 2020; 66(2): e-10996.

18. MINISTÉRIO DA SAÚDE. Consenso Nacional de Nutrição Oncológica, 2015. Disponível em: https://www.inca.gov.br/sites/ufu.sti.inca.local/files/media/document/consenso-nacional-de-nutricao-oncologica-2edicao-2015.pdf. Acessado em: 09 de fevereiro de 2022.

19. MINISTÉRIO DA SAÚDE. Consenso Nacional de Nutrição Oncológica Volume II, 2016. Disponível em: https://www.inca.gov.br/sites/ufu.sti.inca.local/files/media/document/consenso-nutricao-oncologica-vol-ii-2-ed2016.pdf. Acessado em: 09 de fevereiro de 2022.

20. MINISTÉRIO DA SAÚDE. Estimativa 2020: Incidência de Câncer no Brasil, 2019. Disponível em: https://www.inca.gov.br/publicacoes/livros/estimativa-2020-incidencia-de-cancer-no-brasil. Acessado em: 09 de fevereiro de 2022.

21. PAULA ALB, et al. Frequência de desnutrição pela espessura do músculo adutor do polegar em pacientes com câncer de cabeça e pescoço. Trabalho de Conlusão de Residência (Residência em Atenção em Oncologia) - Universidade Federal de Uberlândia, Uberlândia, 2019; 16 p.

22. POZIOMYCK AK, et al. Métodos de avaliação nutricional preditores de mortalidade pós-operatória em pacientes submetidos à gastrectomia por câncer gástrico. Revista do Colegio Brasileiro de Cirurgioes, 2017; 44(5): 482-490.

23. POZIOMYCK AK, et al. Espessura do músculo adutor do polegar e predição de mortalidade pós-operatória em pacientes com câncer gástrico. Arquivos Brasileiros de Cirurgia Digestiva, 2018; 31(1): 1-4.

24. RODRIGUES BC, et al. Avaliação do Risco Nutricional em Pacientes Onco-Hematológicos Hospitalizados. Revista Brasileira de Cancerologia, 2019; 65(1): e-01266.

25. SCHLÜSSEL MM, et al. Hand grip strength test and its use in nutritional assessment. Rev Nutr, 2008; 21(2): $223-235$.

26. SILVA S, PINHO J. Adaptação transcultural e validação da versão em português da Pontuação de Avaliação Subjetiva Global Gerada pelo Paciente (PG-SGA). Clinical Nutrition, 2015; 34(S1): S194-S195.

27. SOCIEDADE BRASILEIRA DE NUTRIÇÃO ONCOLÓGICA. I Consenso Brasileiro de Nutrição Oncológica, 2021. Disponível em: https://www.sbno.com.br/wp-content/uploads/2021/07/consenso_2021.pdf. Acessado em: 11 de fevereiro de 2022.

28. STEEMBURGO T, et al. Hand Grip Strength and nutritional status in hospitalized oncological patients. Revista de Nutricao, 2018; 31(5): 489-499.

29. VALE IAV, et al. Avaliação e Indicação Nutricional em Pacientes Oncológicos no Início do Tratamento Quimioterápico. Revista Brasileira de Cancerologia, 2015; 61(4): 367-372.

30. VALENTE KP, et al. Association of Adductor Pollicis Muscle Thickness and Handgrip Strength with nutritional status in cancer patients. Plos One, 2019; 14(8): 1-12.

31. VALMORBIDA A, et al. Acceptance of an adapted ice cream as dessert by onco-hematological patients. Nutricion Clinica y Dietetica Hospitalaria, 2019; 39(2): 148-155.

32. WORLD HEALTH ORGANIZATION (WHO). Physical status: the use and interpretation of anthropometry. 1993. Disponível em: https://apps.who.int/iris/bitstream/handle/10665/37003/WHO_TRS_854.pdf?sequence=1\&isAllowed=y 
. Acessado em: 09 de fevereiro de 2022. 